\title{
Ten minutes endurance test at $50 \%$ upper body weight: a pilot study in healthy controls and physically active people
}

Christoph Anders ( $\nabla$ christoph.anders@med.uni-jena.de)

Universitätsklinikum Jena: Universitatsklinikum Jena https://orcid.org/0000-0002-5580-5338

Tim Schönau

Universitätsklinikum Jena: Universitatsklinikum Jena

\section{Research note}

Keywords: Endurance capacity, back muscles, training modalities

Posted Date: July 9th, 2021

DOl: https://doi.org/10.21203/rs.3.rs-693065/v1

License: (c) (i) This work is licensed under a Creative Commons Attribution 4.0 International License.

Read Full License 


\section{Abstract}

\section{Objective}

Intense endurance and strength training mark virtually opposite parts of basic motor skills. Extreme high load physical demands are getting sparer but endurance demands are still present. Therefore, healthy controls and endurance and strength trained athletes at competition level were exposed to a submaximal endurance test of their back muscles.

In this pilot study 38 healthy male subjects participated: physically inactive controls $(C, n=12)$, endurance trained $(E T, n=13)$, and strength trained subjects $(S T, n=13)$. All participants were asked to finish a ten minutes back muscle endurance test at $50 \%$ of their upper body weight.

\section{Results}

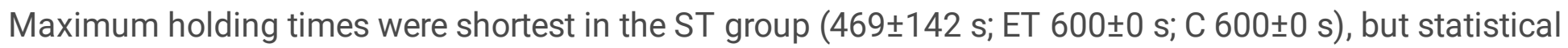
significance could only be proven for ET vs. ST $(p<0.01)$. Hedges gs values for comparisons of maximum holding times showed relevant differences among all groups: ET vs. ST 10.64; ET vs. C 2.38; ST vs. C 0.78. Values of perceived exertion increased over time with lowest values for the ET group, except after the first minute. Especially between 180 s and 420 s ST group showed highest exertion values, but between group differences could not be determined.

\section{Introduction}

To counteract today's inactive lifestyle, recreational sports is becoming increasingly popular, since positive effects on metabolic processes [1], or more generally life satisfaction especially in old age [2] can be identified to name just two examples. Nowadays, there is a wide range of possibilities, whereby every physical activity can ultimately be traced back to the basic motor skills strength, endurance, speed, agility and coordination. Depending on the chosen training modality, the respective training focus is either on several aspects at the same time or individual aspects are particularly emphasized. In particular, strength and endurance are possible isolated individual training goals, while speed, agility and coordination can be regarded as a prerequisite for any type of sports activity in terms of training progress and protection against injury. However, the maintenance or training of these basic skills plays a role not only for athletic activity, but also for everyday life. Here we must react appropriately to a wide variety of physical demands. Since extreme strength requirements in everyday life are becoming sparer, endurance training in particular represents an essential part of everyday physical demands. The impact of extreme training intensities of endurance and strength training was therefore investigated in the present pilot study. For this investigation inactive control subjects as well as endurance and strength athletes were exposed to a ten-minute endurance test of their back muscles at $50 \%$ of their upper body weight.

\section{Methods}


For this pilot study we recruited 38 healthy male participants. Participants consisted of three subgroups: a group of physically inactive people (Control (C), $n=12$ ) and two groups of physically active people at competition level (at least four training sessions per week). The two physically active groups practised either endurance (ET, cycling and triathlon, $n=13$ ) or strength training (ST, power lifting, $n=13$ ). Participants were informed about the procedure and aim of the study and signed informed consent to voluntary participate in this investigation. The study was approved by the ethics committee of the Friedrich-Schiller University Jena (2020-1844-BO). Details about the study participants are provided in Table 1.

Table 1

Participant characteristics

\begin{tabular}{|llll|}
\hline & Endurance & Strength & Control \\
\hline Age [years] & $22.2 \pm 2.9$ & $23.5 \pm 1.8$ & $22.1 \pm 1.0$ \\
\hline Height [cm] & $184 \pm 6.5$ & $180 \pm 5.6$ & $184 \pm 5.9$ \\
\hline Weight $[\mathrm{kg}]$ & $72.8 \pm .7 .0$ & $90.3 \pm 13.9 \S \S$ & $78.9 \pm .14 .3$ \\
\hline BMI [kg/m²] & $21.5 \pm 1.3$ & $27.7 \pm 3.8 \S \S$ & $23.1 \pm .3 .8 \$$ \\
\hline UBW [kg] & $31.7 \pm 2.8$ & $37.0 \pm 4.5 \S$ & $32.7 \pm 4.5$ \\
\hline UBT [Nm] & $123 \pm 12.3$ & $134 \pm 21.8$ & $125 \pm 19.5$ \\
\hline BMI: body mass index; UBW: upper body weight; UBT: upper body torque \\
\hline
\end{tabular}

$\S p<0.05$ vs. endurance; $\S \S p<0.01$ vs. endurance; $\$ p<0.05$ vs. strength

Standing in upright posture participants were positioned in a computerized test and training device (CTT Centaur, BfMC, Leipzig, Germany). In this device the subjects' lower body is fixed while the upper body maintains freedom of movement. The device is equipped with a harness, positioned over the subjects' shoulder that contains strain gauges for force measurement in frontal and sagittal directions. Additionally the device is equipped with a small feedback monitor in front of the subject. Here a moving target point is displayed that is located in the centre of a crosshair if not net force acts on the harness, i.e. the subject maintains upright body posture. By tilting the device up to horizontal position gravitational forces act on the trunk, enabling defined load levels up to $100 \%$ of the subject's upper body weight (UBW). For warm up participants completed a set of submaximal flexion and extension tests. Subsequently they were exposed to $50 \%$ UBW extension load by tilting them forward at an angle of $30^{\circ}$. For this endurance test a maximum of $600 \mathrm{~s}$ was defined as the target time. Participants were requested to maintain their upright position until target time was reached, or until exhaustion. After every completed minute they were asked about their level of perceived exertion by using the Borg scale [3]. 
To test differences of maximum holding times among groups basically t-tests were applied, together with the respective Bonferroni-correction for multiple testing. In addition, effect sizes were calculated to address relevance of differences. For this we used Hedges gs as it is particularly suited for small sample sizes [4]. To assess differences of the Borg values between groups the non-parametric Kruskal and Wallis test was applied, followed by the respective post hoc tests.

\section{Results}

Maximum holding times were shortest for ST (Fig. 1), but showed significant differences only for ET vs. ST $(p<0.01)$. Anyhow, Hedges gs values showed relevant differences among all groups: ET vs. ST 10.64; ET vs. C 2.38; ST vs. C 0.78 . Values of perceived exertion increased over time with lowest values for the ET group, except after $60 \mathrm{~s}$ (Fig. 2). Especially between 180s and 420s ST group showed highest exertion values. Although the non-parametric Kruskal-Wallis $\mathrm{H}$ test showed significant differences among the groups at $180,240,300,420$, and 600 s post hoc tests failed to verify significant group differences.

\section{Discussion}

The study revealed a clear advantage of endurance trained but also untrained subjects over strength trained athletes with respect to their back muscle endurance capacity at 50\% UBW force level. Further, all athletes were highly competitive, thus any premature termination of the endurance task can be excluded with high certainty. Thus, the absolute as well as relative strength level of back muscles seems to be rather inversely correlated with endurance performance. The lower limit, i.e. values for persons with an even lower strength reserve, could not be determined on basis of the available data. Anyhow, it can be assumed that back muscles endurance performance follows a U-shaped curve, i.e. deficits in endurance performance are expected to be observed in persons with low strength reserve as well. There are indirect indications for this assumption, since values of perceived exertion were always lowest in the ET group, whereas the control group showed highest values after $480 \mathrm{~s}$ endurance time, unfortunately without statistical prove. However, the Borg values of the control group may also be caused by lack of experience with respect to physical exertion in general. This is particularly evident for the value after $60 \mathrm{~s}$, where the control group tended to have the lowest values with a mean value of 9 , while the two groups of athletes each reported values of 10 . Here it would make sense to carry out a repeated examination shortly after this initial examination to test whether the assessment of perceived exertion changes.

We did not take muscle biopsies here but it can be expected that back muscles fibre composition between the two competitive athlete groups differed significantly $[5,6,7]$. For the ST group a largely higher proportion of Type II fibres can be assumed in comparison with the ET group and also larger fibre diameters [8], resulting in early use of Type II fibres and reduced metabolic supply due to the enlarged fibre diameters.

\section{Limitations}


- Only few subjects were investigated, therefore the results are preliminary

- Premature termination due to motivational reasons cannot completely be excluded

- Results were gathered during a defined, but artificial test situation, therefore the results cannot be transferred directly to everyday endurance tasks

\section{Abbreviations}

\begin{tabular}{|ll|}
\hline Unabbreviated term & abbreviation \\
\hline Control & C \\
\hline Endurance trained & ET \\
\hline Strength trained & ST \\
\hline Body mass index & BMI \\
\hline Upper body weight & UBW \\
\hline Upper body torque & UBT \\
\hline
\end{tabular}

\section{Declarations}

\section{Ethics approval and consent to participate}

This study was approved by the ethics committee of the Friedrich-Schiller-University Jena. The committee's reference number is 2020-1844-BO. Prior to study participation all participants were informed about the purpose and course of the study and signed an informed consent form.

\section{Consent for publication}

Not applicable.

\section{Availability of data and materials}

The dataset supporting the conclusions of this article is included in an additional supplementary datafile.

\section{Competing interests}

The authors declare that they have no competing interests.

\section{Funding}




\section{Authors' contributions}

CA made substantial contributions to the conception and design of the work, acquisition, analysis, interpretation of data, has drafted the work, approved the submitted version, and has agreed both to be personally accountable for the author's own contributions and to ensure that questions related to the accuracy or integrity of any part of the work, even ones in which the author was not personally involved, are appropriately investigated, resolved, and the resolution documented in the literature.

TS made substantial contributions to acquisition, analysis, interpretation of data, approved the submitted version, and has agreed both to be personally accountable for the author's own contributions and to ensure that questions related to the accuracy or integrity of any part of the work, even ones in which the author was not personally involved, are appropriately investigated, resolved, and the resolution documented in the literature.

\section{Acknowledgements}

The authors want to thank all participants for their time and effort they spent for this investigation.

\section{References}

1. Barbosa JPDS, Basso L, Seabra A, et al. Relationship between physical activity, physical fitness and multiple metabolic risk in youths from Muzambinho's study. Eur J Sport Sci. 2016;16:618-23. doi:https://doi.org/10.1080/17461391.2015.1088576.

2. Cho D, Post J, Kim SK. Comparison of passive and active leisure activities and life satisfaction with aging. Geriatr Gerontol Int. 2018;18:380-6. doi:10.1111/ggi.13188.

3. Borg G. Perceived exertion as an indicator of somatic stress. Scand J Rehabil Med. 1970;2:92-8.

4. Lakens $D$. Calculating and reporting effect sizes to facilitate cumulative science: a practical primer for t-tests and ANOVAs. Front Psychol 2013; 4. doi:10.3389/fpsyg.2013.00863.

5. Beck TW, Housh TJ, Fry AC, et al. The influence of muscle fiber type composition on the patterns of responses for electromyographic and mechanomyographic amplitude and mean power frequency during a fatiguing submaximal isometric muscle action. Electromyogr Clin Neurophysiol. 2007;47:221-32.

6. Sarzynski MA, Bouchard C. World-class athletic performance and genetic endowment comment. Nat Metab. 2020;2:796-8. doi:10.1038/s42255-020-0233-6.

7. Simoneau JA, Bouchard C. Genetic Determinism of Fiber-Type Proportion in Human Skeletal-Muscle. Faseb J. 1995;9:1091-5. doi:https://doi.org/10.1096/fasebj.9.11.7649409. 
8. Clarkson PM, Kroll W, McBride TC. Maximal isometric strength and fiber type composition in power and endurance athletes. Eur J Appl Physiol Occup Physiol. 1980;44:35-42. doi:https://doi.org/10.1007/BF00421761.

\section{Figures}

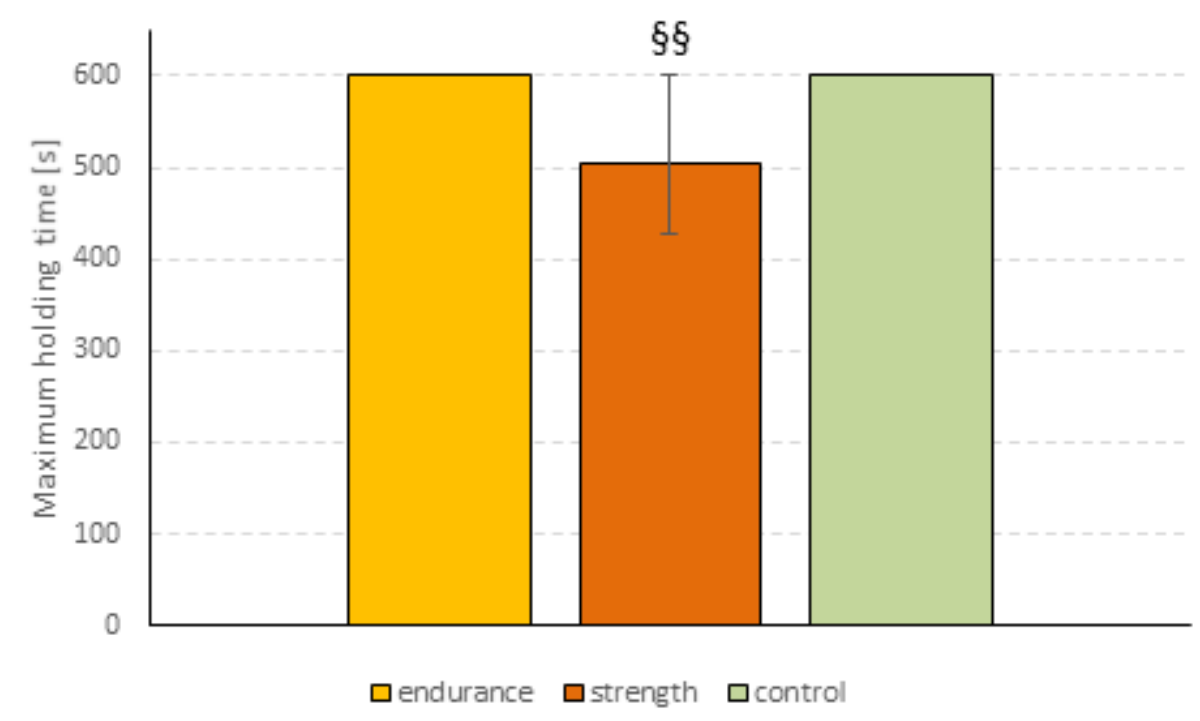

$\S \S p \leq 0.01$ vs. endurance

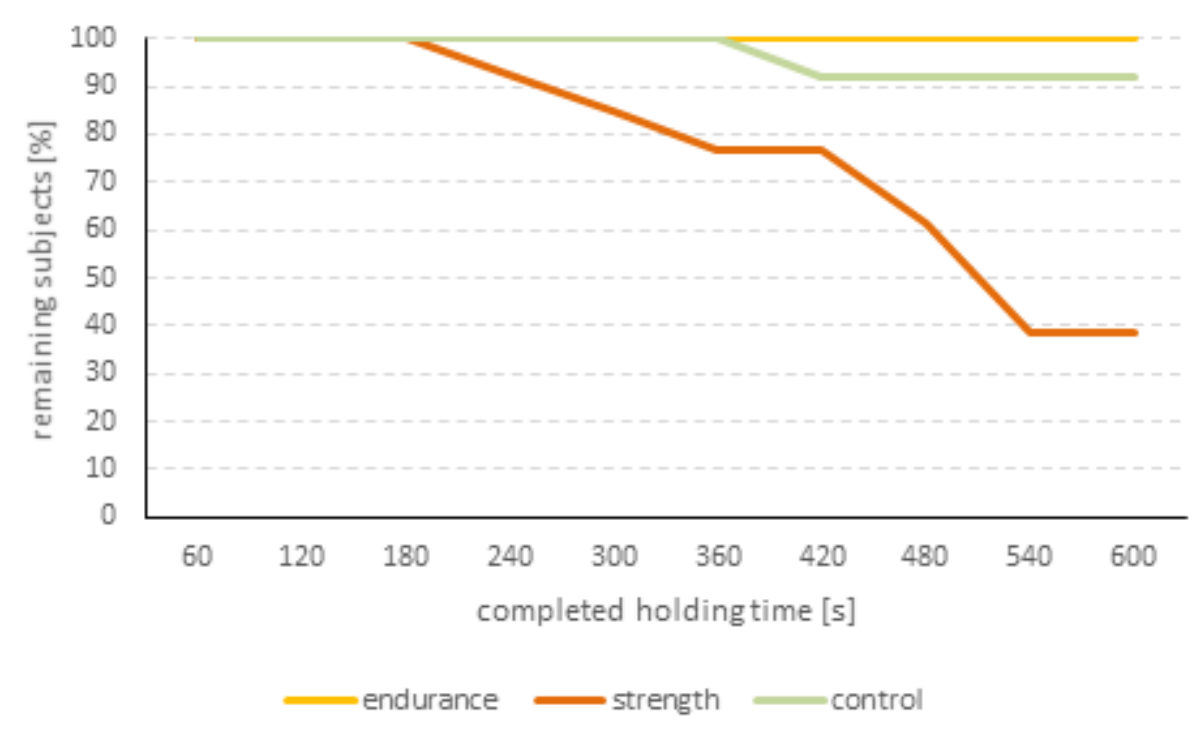

\section{Figure 1}

Maximum endurance times (upper panel) and remaining subjects (lower panel) for the endurance task. Values are given as mean values \pm SD. Statistical differences are indicated. 


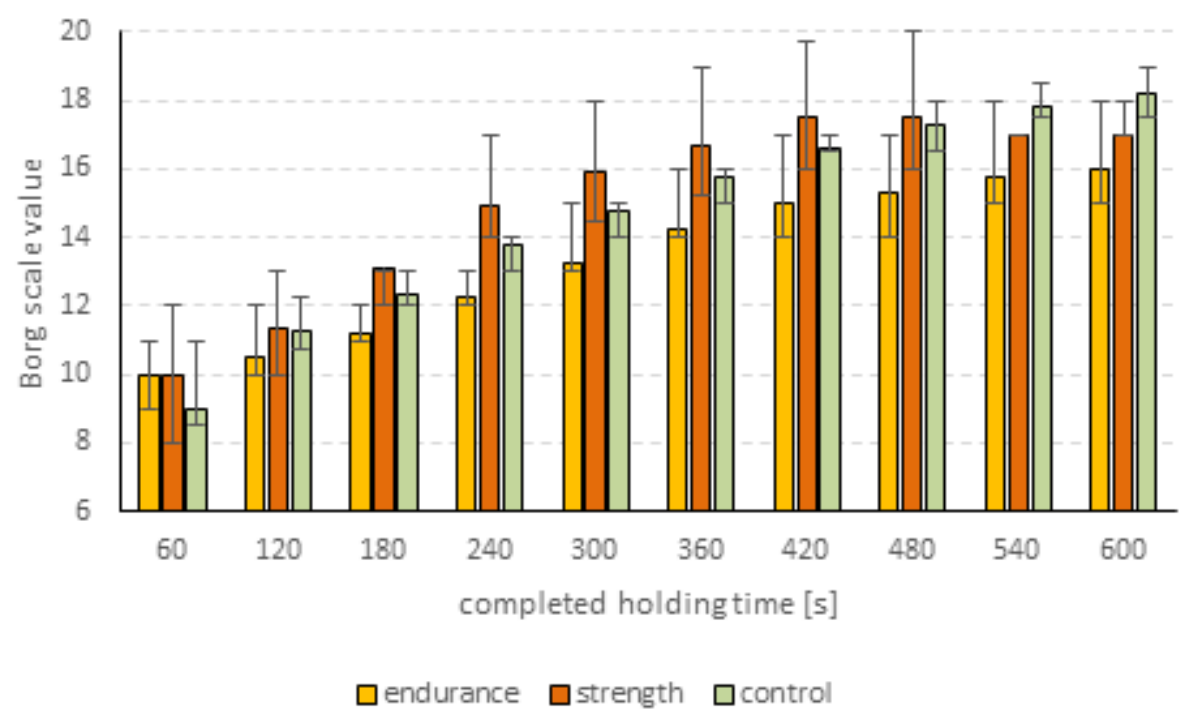

Figure 2

Borg scale values during the endurance task Values are given as median values \pm quartiles. No statistical differences could be detected between groups.

\section{Supplementary Files}

This is a list of supplementary files associated with this preprint. Click to download.

- studydatabackmuscleendurance.xlsx 